\title{
Multi-criteria decision-making method with double risk parameters in interval-valued intuitionistic fuzzy environments
}

\author{
Li-li Niu ${ }^{1}$ Jian $\mathrm{Li}^{2}$ (i) $\cdot$ Feilong $\mathrm{Li}^{3} \cdot$ Zhong-xing Wang $^{4}$
}

Received: 9 December 2019 / Accepted: 3 June 2020 / Published online: 19 June 2020

(c) The Author(s) 2020

\begin{abstract}
In the multi-criteria decision-making (MCDM) process, decision-makers with different risk attitudes may have different decision results. To address this issue and present decision-makers' mentality, this paper introduces two mentality parameters. These parameters reflect the decision-makers' risk attitudes in determining the membership and non-membership degrees of the evaluation information. In addition, the parameters demonstrate the risk attitude in terms of the hesitancy degree under interval-valued intuitionistic fuzzy information. Then, a new score function of interval-valued intuitionistic fuzzy numbers (IVIFNs) is proposed that uses the introduced mentality parameters. Meanwhile, certain properties of the proposed score function are discussed. Furthermore, the weighted comprehensive score value of IVIFNs is introduced, and an MCDM method is developed in an interval-valued intuitionistic fuzzy environment. Finally, a numerical example and comparative analyses are provided to illustrate the feasibility and effectiveness of the proposed method.
\end{abstract}

Keywords Multi-criteria decision-making · Risk attitudes · Interval-valued intuitionistic fuzzy numbers $\cdot$ Score function · Mentality parameter

\section{Introduction}

In 1986, Atanassov [1] proposed intuitionistic fuzzy sets (IFSs), which are an extension of fuzzy sets [2]. In IFSs, the membership, non-membership, and hesitancy degrees of an element that belongs to a set are considered. These new sets are more flexible and practical than traditional fuzzy sets in dealing with the uncertainty of the objectives [3-9]. For example, Melliani and Castillo [7] introduced recent advances in intuitionistic fuzzy logic systems, Roeva and Michalikova [8] proposed a generalized net model based on intuitionistic fuzzy logic control, and Atanassov and

Jian Li

jian2016@csu.edu.cn

1 Guangxi University Xingjian College of Science and Liberal Arts, Nanning 530005, People's Republic of China

2 School of Logistics Management and Engineering, Nanning Normal University, Nanning 530001, People's Republic of China

3 Guangxi Medical University Affiliated Tumor Hospital, Nanning 530021, People's Republic of China

4 School of Mathematics and Information Science, Guangxi University, Nanning 530004, People's Republic of China
Sotirov [9] discussed neural networks with interval valued intuitionistic fuzzy conditions. However, in some complex decision-making situations, decision-makers may not have sufficient knowledge to provide crisp values of membership and non-membership degrees. Nonetheless, their ranges can be indicated. Therefore, in 1989, Atanassov and Gargov [10] introduced the concept of interval-valued intuitionistic fuzzy sets (IVIFSs), whose membership and non-membership are closed intervals instead of crisp values. Because the membership degree and non-membership degree of IVIFSs are described by intervals, scholars have considered IVIFSs as a suitable tool to express uncertain and vague information for practical issues [11-14].

Over the last few decades, IVIFSs have been widely applied in multi-criteria decision-making (MCDM) [15-21], such as the evaluation of service quality in public bus transportation [22], healthcare evaluation in hazardous waste recycling [18], multi-perspective collaborative scheduling [23], evaluation of the risk of failure modes [24], photovoltaic module selection [25], measurement of the service quality of urban rail transit [20], and evaluation of the effectiveness of knowledge transfer [21]. The main goal in solving MCDM problems is to rank the admissible alternatives that have been evaluated based on the given attributes and 
to choose the best one. Thus, with respect to the abovementioned MCDM problems which involve many interval-valued intuitionistic fuzzy number (IVIFN) methods, how to select a reasonable approach to rank IVIFNs becomes an important research topic.

A considerable number of studies have been conducted on ranking and comparing IVIFNs [26-32]. For example, some ranking methods are based on the possibility degree and divergence degree [17], geometric aspect [30, 33], complex proportional assessment method [18], distance-based approach [31, 34-36], score function [26, 37], generalized exponential entropy [32], and probability density functions and variances of IVIFNs [38]. Numerous methods have been developed to rank and compare IVIFNs in the abovementioned literature. These methods can be roughly classified into three categories: (1) independent ranking [17, 26, 32, 37, 39], (2) pairwise comparison-based ranking [40, 41], and (3) reference based ranking [34-36]. The processes have been developed from various perspectives to rank and compare IVIFNs with applications to solve real-life decision making problems.

Among these approaches to rank IVIFNs, the score function is an effective method. Xu and Chen [42] introduced a ranking method based on the score function and accuracy function of IVIFVs. Ye [29] proposed a novel accuracy function for IVIFNs by taking into account the hesitancy degree of IVIFNs. Lakshmana Gomathi Nayagam et al. [43] provided a novel accuracy function for IVIFNs to overcome the difficult decision-making process of existing accuracy functions in some cases. Wang and Chen [26] proposed a new score function of IVIFNs to handle the drawback of Chen and Huang [44] method. In another study, Wang and Chen [27] further introduced the new score function and accuracy function of IVIFNs to deal with the drawback of Wang and Chen [26] method. Nguyen [45] defined a generalized p-norm knowledge-based score function for IVIFNs, which is a generalization of the score function of IFSs. Additional score functions of IVIFNs can be found in [46, 47].

These methods are effective in solving decision-making problems in interval-valued intuitionistic fuzzy environments. However, the methods suffer from several shortcomings. (1) The ranking method cannot distinguish whether two IVIFNs have equal middles of membership degree and nonmembership intervals [42] or have equal accuracy functions $[29,43]$. (2) In the decision-making process, the decision result depends on the risk attitude of decision-makers. For example, risk-averse decision-makers are inclined to select low-risk alternatives, whereas risk-seeking decision-makers tend to select high-risk options. However, there are few studies on these aspects among most of the abovementioned ranking methods. (3) Some methods neglect the discussion of a reasonable score function, which can generate unreasonable and unreliable decision results [17].
To overcome these shortcomings, the objectives of this study are summarized as follows:

1. To show that the ranking results are strongly affected by the decision-makers' risk attitudes, this study introduces two mentality parameters.

2. A new score function of IVIFNs is proposed by introducing mentality parameters and integrating the membership, non-membership, and hesitancy degrees of IVIFNs.

3. Some basic properties of the proposed score function of IVIFNs are discussed to verify its reasonability and effectiveness, which can make the decision results believable and trustworthy.

The remainder of the paper is organized as follows. In "Preliminaries", we briefly review basic concepts such as IFSs and IVIFSs. In "New score function of IVIFNs", a new score function is proposed based on the discussion of the hesitation of the IVIFNs to properly reflect the decisionmakers' risk attitudes. Several illustrative examples are provided to show the feasibility of the proposed method. In "MCDM in interval-valued intuitionistic fuzzy environments", we apply the proposed score function to MCDM with IVIFNs. In "Illustrative example", an illustrative example and comparative analyses are provided to demonstrate the practicality and effectiveness of our method. The conclusions are presented in "Conclusions".

\section{Preliminaries}

This section presents the basic concepts of IFSs and IVIFSs.

Definition 1 [1] Let $X$ be a fixed set with a nonempty universe. An IFS $A$ in $X$ is denoted as $A=\left\{\left\langle x, \mu_{A}(x), v_{A}(x)\right\rangle \mid x \in X\right\}$, where $\mu_{A}(x) \in[0,1]$ and $v_{A}(x) \in[0,1]$ represent the membership and non-membership degrees of element $x$ to set $A$, respectively, satisfying $0 \leq \mu_{A}(x)+v_{A}(x) \leq 1$ for all $x \in X$. The pair $\left(\mu_{A}(x), v_{A}(x)\right)$ is called an intuitionistic fuzzy number.

The concept of IFSs is further extended to IVIFSs.

Definition 2 [10] Let $X$ be a finite and non-empty set. An IVIFS $\tilde{A}$ in $X$ is an object with the following form.

$\tilde{A}=\left\{\left\langle x, \mu_{\tilde{A}}(x), v_{\tilde{A}}(x)\right\rangle \mid x \in X\right\}$,

where the functions $\mu_{\tilde{A}}(x): X \rightarrow D[0,1]$ and $v_{\tilde{A}}(x)$ : $X \rightarrow D[0,1]$ denote the membership degree and non-membership degree of element $x \in X$ in $\tilde{A}$, respectively. The pair $\left(\mu_{\tilde{A}}(x), v_{\tilde{A}}(x)\right)$ is called an IVIFN. $\mu_{\tilde{A}}(x)$ and $v_{\tilde{A}}(x)$ are two closed intervals, and their lower and upper boundaries are 
denoted by $\mu_{\tilde{A} L}(x), \mu_{\tilde{A} U}(x), v_{\tilde{A} L}(x)$, and $v_{\tilde{A} U}(x)$, respectively. IVIFS $\tilde{A}$ is denoted as

$\tilde{A}=\left\{\left\langle x,\left[\mu_{\tilde{A} L}(x), \mu_{\tilde{A} U}(x)\right],\left[\nu_{\tilde{A} L}(x), \nu_{\tilde{A} U}(x)\right]\right\rangle \mid x \in X\right\}$,

where $0 \leq \mu_{\tilde{A} U}(x)+v_{\tilde{A} U}(x) \leq 1, \mu_{\tilde{A} L}(x) \geq 0$, and $v_{\tilde{A} L}(x) \geq 0$. Specifically, if $\mu_{\tilde{A} L}(x)=\mu_{\tilde{A} U}(x)$ and $v_{\tilde{A} L}(x)=v_{\tilde{A} U}(x)$, then IVIFS $\tilde{A}$ reduces to an IFS.

For simplicity, IVIFN $\tilde{\alpha}=\left(\mu_{\tilde{\alpha}}(x), v_{\tilde{\alpha}}(x)\right)$ is usually denoted as $\tilde{\alpha}=([a, b],[c, d])$, where $[a, b] \subset D[0,1]$, $[c, d] \subset D[0,1]$, and $b+d \leq 1$.

Example 1 Let $\tilde{\alpha}=([0.6,0.7],[0.1,0.2])$ be an IVIFN; its meaning can be explained as follows: for an election with 100 voters, the value $[0.6,0.7]$ implies that $60-70$ are expected to be in favor, the value $[0.1,0.2]$ implies that $10-20$ are expected to be against, and the value $[0.1,0.3]$ implies that $10-30$ are expected to abstain.

Definition 3 [10] Let $\tilde{\alpha}=\left(\mu_{\tilde{\alpha}}(x), v_{\tilde{\alpha}}(x)\right)$ be an IVIFN.

$$
\begin{aligned}
\pi_{\tilde{\alpha}}(x) & =1-\mu_{\tilde{\alpha}}(x)-v_{\tilde{\alpha}}(x) \\
& =\left[1-\mu_{\tilde{\alpha} U}(x)-v_{\tilde{\alpha} U}(x), 1-\mu_{\tilde{\alpha} L}(x)-v_{\tilde{\alpha} L}(x)\right],
\end{aligned}
$$

then $\pi_{\tilde{\alpha}}(x)$ is called the hesitancy degree of $\tilde{\alpha}$.

$\mathrm{Xu}$ [48] introduced some basic arithmetical operations of IVIFNs as follows.

Definition 4 [48] Let $\tilde{\alpha}=\left(\left[a_{1}, b_{1}\right],\left[c_{1}, d_{1}\right]\right)$ and $\tilde{\beta}=\left(\left[a_{2}, b_{2}\right],\left[c_{2}, d_{2}\right]\right)$ be two IVIFNs. Then, their operational laws are defined as:

$\tilde{\alpha}>\tilde{\beta}$ if $a_{1}>a_{2}, b_{1}>b_{2}, c_{1}<c_{2}$ and $d_{1}<d_{2} ;$

$\tilde{\alpha} \sim \tilde{\beta}$ if $a_{1}=a_{2}, b_{1}=b_{2}, c_{1}=c_{2}$, and $d_{1}=d_{2}$.

\section{New score function of IVIFNs}

In this section, we review several representative score functions of IVIFNs and discuss their shortcomings. This is our motivation for proposing a new score function of IVIFNs, which is provided in "New score function of IVIFNs".

For simplicity, we use the following symbols: let $\tilde{\alpha}=([a, b],[c, d])$ be an IVIFN, $M\left(\mu_{\tilde{\alpha}}\right)=\frac{a+b}{2}$ denotes the middle value of the membership interval, $M\left(v_{\tilde{\alpha}}\right)=\frac{c+d}{d}$ is the non-membership interval, and $M\left(\pi_{\tilde{\alpha}}\right)=\frac{(1-b-\tilde{d})+(1-a-c)}{2}$ is the hesitancy interval, where $M\left(u_{\tilde{\alpha}}\right)+M\left(v_{\tilde{\alpha}}\right)+M\left(\pi_{\tilde{\alpha}}\right)=1$.

\section{Some representative score functions of IVIFNs}

$\mathrm{Xu}$ and Chen [42] introduced the score function and accuracy function of IVIFNs as follows.

Definition 5 [42] For an IVIFN $\tilde{\alpha}=([a, b],[c, d])$, the score function and accuracy function can be denoted as:

$S(\tilde{\alpha})=\frac{a-c+b-d}{2}$,

$h(\tilde{\alpha})=\frac{a+b+c+d}{2}$.

Obviously, $S(\tilde{\alpha})=M\left(u_{\tilde{\alpha}}\right)-M\left(v_{\tilde{\alpha}}\right) \in[-1,1], \quad$ and $h(\tilde{\alpha})=M\left(u_{\tilde{\alpha}}\right)+M\left(v_{\tilde{\alpha}}\right) \in[0,1]$. The larger the score value of $\tilde{\alpha}$ is, the larger IVIFN $\tilde{\alpha}$ is. Based on Definition 5, a prioritized comparison method of IVIFNs is introduced as follows.

Definition 6 [42] For any two IVIFNs $\tilde{\alpha}$ and $\tilde{\beta}$,

1. If $S(\tilde{\alpha})<S(\tilde{\beta})$, then $\tilde{\alpha}$ is smaller than $\tilde{\beta}$, denoted as $\tilde{\alpha} \prec \tilde{\beta}$;

2. If $S(\tilde{\alpha})>S(\tilde{\beta})$, then $\tilde{\alpha}$ is larger than $\tilde{\beta}$, denoted as $\tilde{\alpha}>\tilde{\beta}$;

3. If $S(\tilde{\alpha})=S(\tilde{\beta})$, then

(1) if $h(\tilde{\alpha})<h(\tilde{\beta})$, then $\tilde{\alpha}$ is smaller than $\tilde{\beta}$, denoted as $\tilde{\alpha} \prec \tilde{\beta}$;

(2) if $h(\tilde{\alpha})>h(\tilde{\beta})$, then $\tilde{\alpha}$ is larger than $\tilde{\beta}$, denoted as $\tilde{\alpha}>\tilde{\beta}$;

(3) if $h(\tilde{\alpha})=h(\tilde{\beta})$, then $\tilde{\alpha}$ is equivalent $\tilde{\beta}$, denoted as $\tilde{\alpha} \sim \tilde{\beta}$.

The score function and accuracy function are considered at the midpoint of the membership interval and non-membership interval of the IVIFN. Most of the order relationship of IVIFNs can be distinguished by using this method. Unfortunately, we cannot identify the optimal method when the midpoints are equal by applying Definition 6. This shortcoming is shown in Example 2.

Example 2 Let $\tilde{\alpha}_{1}=([0.3,0.5],[0.1,0.3])$ and $\tilde{\alpha}_{2}=([0.4,0.4],[0.2,0.2])$ be two IVIFNs for two alternatives.

Via Eq. (5), we obtain $S\left(\tilde{\alpha}_{1}\right)=S\left(\tilde{\alpha}_{2}\right)=0.2$. Then, we obtain $h\left(\tilde{\alpha}_{1}\right)=h\left(\tilde{\alpha}_{2}\right)=0.6$ by applying Eq. (6). In this case, the order relationship of $\tilde{\alpha}_{1}$ and $\tilde{\alpha}_{2}$ cannot be distinguished.

Considering the limitations of Xu's method, Ye [29] and Lakshmana Gomathi Nayagam et al. [43] introduced new accuracy functions by considering the hesitancy degree of the IVIFN. 
Definition 7 [29] Let $\tilde{\alpha}=([a, b],[c, d])$ be an IVIFN. Its accuracy function is denoted as:

$J(\tilde{\alpha})=a+b-1+\frac{c+d}{2}$.

Definition 8 [43] Let $\tilde{\alpha}=([a, b],[c, d])$ be an IVIFN. Its accuracy function is denoted as:

$L(\tilde{\alpha})=\frac{1}{2}[a+b-d(1-b)-c(1-a)]$.

Ye [29] and Nayagam et al. [43] have asserted that the order relationship of IVIFNs can be obtained using the value of the accuracy function. Unfortunately, these methods have some shortcomings.

Example 3 Let $\tilde{\alpha}_{3}=([0.1,0.2],[0.6,0.8])$ and $\tilde{\alpha}_{4}=([0.4,0.5],[0,0.1])$ be two IVIFNs for two alternatives.

Since the membership degree interval of $\tilde{\alpha}_{3}$ is smaller than that of $\tilde{\alpha}_{4}$, the non-membership degree interval of $\tilde{\alpha}_{3}$ is larger than that of $\tilde{\alpha}_{4}$. According to Eq. (3), we can obtain $\tilde{\alpha}_{3} \prec \tilde{\alpha}_{4}$. This conclusion is intuitive.

In addition, by applying Eq. (7), we obtain $J\left(\tilde{\alpha}_{3}\right)=0$ and $J\left(\tilde{\alpha}_{4}\right)=-0.05$. Since $J\left(\tilde{\alpha}_{3}\right)>J\left(\tilde{\alpha}_{4}\right)$, we have $\tilde{\alpha}_{3}>\tilde{\alpha}_{4}$. The above discussion demonstrates that the order relationship of $\tilde{\alpha}_{3}>\tilde{\alpha}_{4}$ is unreasonable and counterintuitive.

Example 4 Let $\tilde{\alpha}_{5}=([0.3,0.3],[0.2,0.2])$ and $\tilde{\alpha}_{6}=([0.2,0.4],[0.2,0.2])$ be two IVIFNs for two alternatives.

By applying Eq. (8), we obtain $L\left(\tilde{\alpha}_{5}\right)=L\left(\tilde{\alpha}_{6}\right)=0.4$. In this case, the order relationship of alternatives cannot be distinguished. Since the range of the membership degree interval $\tilde{\alpha}_{5}$ is smaller than that of $\tilde{\alpha}_{6}$ and their nonmembership degree intervals are equivalent, the order relationship $\tilde{\alpha}_{5}>\tilde{\alpha}_{6}$ can be easily obtained. This result indicates that the method proposed by Lakshmana Gomathi Nayagam et al. [43] has some shortcomings.

The score function and accuracy function mentioned above are used to obtain the order relationship of IVIFNs. However, these ranking methods do not give sufficient attention to the score function and accuracy function. To overcome these shortcomings, a new method to rank IVIFNs considering the risk attitude of the decision-makers is proposed in the following section.

\section{New score function of IVIFNs}

In the decision-making process, the decision result depends on the risk attitude of the decision-makers. In other words, the risk attitudes of the decision-makers affect the decision results. In this paper, the risk parameter with a new score function of IVIFNs is proposed. Some related properties are discussed to verify the reasonability and effectiveness of the proposed score function.

Before introducing the new score function of IVIFN, we first introduce $\lambda \in[0,1]$ to reflect the decisionmakers' risk attitude in determining the membership degree and the non-membership degree. For an IVIFN $\tilde{\alpha}=([a, b],[c, d])$, let $M^{\lambda}\left(u_{\tilde{\alpha}}\right)=a+\lambda(b-a)$ and $M^{\lambda}\left(v_{\tilde{\alpha}}\right)=c+(1-\lambda)(d-c)$. Then, the hesitancy degree integrated with decision-makers' risk attitudes can be obtained as follows: $M^{\lambda}\left(\pi_{\tilde{\alpha}}\right)=1-M^{\lambda}\left(u_{\tilde{\alpha}}\right)-M^{\lambda}\left(v_{\tilde{\alpha}}\right)$ $=\lambda(1-b-c)+(1-\lambda)(1-a-d) . \quad$ C 1 e a r $1 \mathrm{y}$, we have $M^{\lambda}\left(u_{\tilde{\alpha}}\right) \in[a, b], \quad M^{\lambda}\left(v_{\tilde{\alpha}}\right) \in[c, d]$, and $M^{\lambda}\left(\pi_{\tilde{\alpha}}\right) \in[1-b-d, 1-a-c]$. The value of $\lambda$ depends on the risk attitude of the decision-makers. If $0 \leq \lambda<1 / 2$, the decision-makers are risk-averse. If $\lambda=1 / 2$, the decisionmakers are risk-neutral. If $1 / 2<\lambda \leq 1$, the decision-makers are risk-seeking.

For the hesitancy interval according to the vote model, supposing that people with hesitancy are always affected by supporters and opponents, which lead to support and opposition, respectively, we introduce the second attitude parameter $\theta \in[0,1]$. The attitude parameter $\theta$ expresses the proportion of the supporters in the original group of people with hesitancy. Among those with hesitancy, $\theta M^{\lambda}\left(\pi_{\tilde{\alpha}}\right)$ tend to support, whereas $(1-\theta) M^{\lambda}\left(\pi_{\tilde{\alpha}}\right)$ tend to oppose. If $0 \leq$ $\theta<1 / 2$, the decision-makers are risk-averse. The smaller the value $\theta$ is, the more risk-averse the decision-makers are. If $1 / 2<\theta \leq 1$, the decision-makers are risk-seeking. The larger the value $\theta$ is, the more risk-seeking the decisionmakers are. If $\theta=1 / 2$, the decision-makers are risk-neutral.

Combining these demonstrations, the new score function of IVIFNs is defined as follows.

Definition 9 Let $\tilde{\alpha}=([a, b],[c, d])$ be an IVIFN. Its score function can be denoted as:

$S_{N e w}(\tilde{\alpha})=\left[M^{\lambda}\left(u_{\tilde{\alpha}}\right)+\theta M^{\lambda}\left(\pi_{\tilde{\alpha}}\right)\right]-\left[M^{\lambda}\left(v_{\tilde{\alpha}}\right)+(1-\theta) M^{\lambda}\left(\pi_{\tilde{\alpha}}\right)\right]$,

where,

$M^{\lambda}\left(u_{\tilde{\alpha}}\right)=a+\lambda(b-a), M^{\lambda}\left(v_{\tilde{\alpha}}\right)=c+(1-\lambda)(d-c)$,

$M^{\lambda}\left(\pi_{\tilde{\alpha}}\right)=\lambda(1-b-c)+(1-\lambda)(1-a-d), \lambda \in[0,1], \theta \in[0,1]$.

This score function can be further simplified as follows:

$S_{\mathrm{New}}(\tilde{\alpha})=M^{\lambda}\left(u_{\tilde{\alpha}}\right)-M^{\lambda}\left(v_{\tilde{\alpha}}\right)+(2 \theta-1) M^{\lambda}\left(\pi_{\tilde{\alpha}}\right)$.

Evidently, if we include $\lambda=\theta=1 / 2$ in Eq. (10), we obtain $S_{\mathrm{New}}(\tilde{\alpha})=S(\tilde{\alpha})$. In this case, the new score function $S_{\mathrm{New}}(\tilde{\alpha})$ proposed in Eq. (10) is equivalent to Xu score function $S(\tilde{\alpha})$ introduced in Eq. (5). This result confirms that 
$\mathrm{Xu}$ and Chen [42] method is a special case of the proposed method.

Example 5 The IVIFNs are identical to those provided in Example 2.

According to Eq. (10), we obtain:

$S_{\mathrm{New}}\left(\tilde{\alpha}_{1}\right)=0.4(2 \theta-1+\lambda)$,

$S_{\text {New }}\left(\tilde{\alpha}_{2}\right)=0.4(2 \theta-1+1 / 2)$.

Then, $S_{\mathrm{New}}\left(\tilde{\alpha}_{1}\right)-S_{\mathrm{New}}\left(\tilde{\alpha}_{2}\right)=0.4(\lambda-1 / 2)$. Therefore, for any $\theta \in[0,1]$, we have the following:

1. If $\lambda<1 / 2$, then $S^{\lambda}\left(\tilde{\alpha}_{1}\right)<S^{\lambda}\left(\tilde{\alpha}_{2}\right)$. Risk-averse decisionmakers believe that $\tilde{\alpha}_{1} \prec \tilde{\alpha}_{2}$;

2. If $\lambda>1 / 2$, then $S^{\lambda}\left(\tilde{\alpha}_{1}\right)>S^{\lambda}\left(\tilde{\alpha}_{2}\right)$. Risk-seeking decision-makers believe that $\tilde{\alpha}_{1}>\tilde{\alpha}_{2}$;

3. If $\lambda=1 / 2$, then $S^{\lambda}\left(\tilde{\alpha}_{1}\right)=S^{\lambda}\left(\tilde{\alpha}_{2}\right)$. Risk-neutral decisionmakers believe that $\tilde{\alpha}_{1} \sim \tilde{\alpha}_{2}$.

The derived rankings may be different due to different risk attitudes of the decision-makers. This result is consistent with the real situation. By using Eq. (7), we obtain that $J\left(\tilde{\alpha}_{1}\right)=J\left(\tilde{\alpha}_{2}\right)=0$. Therefore, we cannot obtain the order relationship of these two alternatives. By applying Eq. (8), we obtain $L\left(\tilde{\alpha}_{1}\right)=0.29$ and $L\left(\tilde{\alpha}_{2}\right)=0.28$. Hence, $\tilde{\alpha}_{1}>\tilde{\alpha}_{2}$, which is consistent with the result if the decision-makers are risk-seeking.

Example 6 The IVIFNs are identical to those provided in Example 3.

According to Eq. (10), we obtain

$S_{\mathrm{New}}\left(\tilde{\alpha}_{3}\right)=-0.7+0.3 \lambda+(2 \theta-1)(0.1+0.1 \lambda)$,

$S_{\text {New }}\left(\tilde{\alpha}_{4}\right)=0.3+0.2 \lambda+0.5(2 \theta-1)$.

Then, $S_{\text {New }}\left(\tilde{\alpha}_{4}\right)-S_{\text {New }}\left(\tilde{\alpha}_{3}\right)=0.6+0.8 \theta-0.2 \lambda \theta>0$. For any $\lambda, \theta \in[0,1]$, we have $S_{\text {New }}\left(\tilde{\alpha}_{4}\right)>S_{\text {New }}\left(\tilde{\alpha}_{3}\right)$, which implies that alternative $\tilde{\alpha}_{4}$ is better than alternative $\tilde{\alpha}_{3}$. This finding is intuitive.

Example 7 The IVIFNs are identical to those provided in Example 4.

According to Eq. (10), we obtain,

$S_{\mathrm{New}}\left(\tilde{\alpha}_{5}\right)=-0.4+\theta$,

$S_{\text {New }}\left(\tilde{\alpha}_{6}\right)=-0.4+\theta+0.4(1-\theta)(\lambda-1 / 2)$.
Then, $\quad S_{\text {New }}\left(\tilde{\alpha}_{6}\right)-S_{\text {New }}\left(\tilde{\alpha}_{5}\right)=0.4(1-\theta)(\lambda-1 / 2)$. Therefore, we obtain the following:

1. If $0 \leq \lambda<1 / 2$ and $0 \leq \theta<1 / 2$, then $S_{\text {New }}\left(\tilde{\alpha}_{5}\right)>S_{\text {New }}\left(\tilde{\alpha}_{6}\right)$. Risk-averse decision-makers believe that $\tilde{\alpha}_{5}>\tilde{\alpha}_{6}$;

2. If $1 \geq \lambda>1 / 2$ and $1>\theta>1 / 2$, then $S_{\text {New }}\left(\tilde{\alpha}_{5}\right)<S_{\text {New }}\left(\tilde{\alpha}_{6}\right)$. Risk-seeking decision-makers believe that $\tilde{\alpha}_{5}<\tilde{\alpha}_{6}$;

3. If $\lambda=1 / 2$ and $\theta=1 / 2$ then, $S_{\text {New }}\left(\tilde{\alpha}_{5}\right)=S_{\text {New }}\left(\tilde{\alpha}_{6}\right)$. Risk-neutral decision-makers believe that $\tilde{\alpha}_{5} \sim \tilde{\alpha}_{6}$.

By using Eqs. (7) and (8), we obtain $\tilde{\alpha}_{5} \sim \tilde{\alpha}_{6}$. The order relationship of these two alternatives cannot be obtained. However, if we use the proposed method, their order relationship can be distinguished when $0 \leq \lambda<1 / 2$ or $1 \geq \lambda>1 / 2$. This finding confirms the effectiveness of the proposed score function.

Example 8 Let $\tilde{\alpha}_{7}=([0.5,0.5],[0.4,0.5])$ and $\tilde{\alpha}_{8}=([0.4,0.4],[0.3,0.4])$ be two IVIFNs for two alternatives.

According to Eq. (10), we obtain,

$S_{\text {New }}\left(\tilde{\alpha}_{7}\right)=0.2 \lambda \theta$,

$S_{\mathrm{New}}\left(\tilde{\alpha}_{8}\right)=0.2 \lambda \theta+0.2(2 \theta-1)$.

Then, $S_{\text {New }}\left(\tilde{\alpha}_{8}\right)-S_{\text {New }}\left(\tilde{\alpha}_{7}\right)=0.2(2 \theta-1)$. For any $\lambda \in[0,1]$, we have the following:

1. If $\theta<1 / 2$, then $S_{N e w}\left(\tilde{\alpha}_{7}\right)>S_{N e w}\left(\tilde{\alpha}_{8}\right)$. Risk-averse decision-makers believe that $\tilde{\alpha}_{7}>\tilde{\alpha}_{8}$;

2. If $\theta>1 / 2$, then $S_{\text {New }}\left(\tilde{\alpha}_{7}\right)<S_{N e w}\left(\tilde{\alpha}_{8}\right)$. Risk-seeking decision-makers believe that $\tilde{\alpha}_{7} \prec \tilde{\alpha}_{8}$;

3. If $\theta=1 / 2$, then $S_{\text {New }}\left(\tilde{\alpha}_{7}\right)=S_{N e w}\left(\tilde{\alpha}_{8}\right)$. Risk-neutral decision-makers believe that $\tilde{\alpha}_{7} \sim \tilde{\alpha}_{8}$.

By using Eqs. (7) and (8), we obtain $\tilde{\alpha}_{7}>\tilde{\alpha}_{8}$, which is consistent with the result of risk-seeking decision-makers.

Some properties of the proposed score function of IVIFN are discussed in the following section to confirm its reasonability and effectiveness.

Property 1. Let $\tilde{\alpha}=([a, b],[c, d])$ be an IVIFN. If the score function $S_{N e w}(\tilde{\alpha})$ is defined by Definition 9 , then.

1. $-1 \leq S_{\mathrm{New}}(\tilde{\alpha}) \leq 1$, for any $\lambda, \theta \in[0,1]$;

2. For any $\lambda, \theta \in[0,1], S_{\mathrm{New}}(\tilde{\alpha})=1$ if and only if $\tilde{\alpha}=([1,1],[0,0])$; 
3. For any $\lambda, \theta \in[0,1], S_{\mathrm{New}}(\tilde{\alpha})=-1$ if and only if $\tilde{\alpha}=([0,0],[1,1])$.

\section{Proof}

1. C o mbining E q $\quad\left(\begin{array}{ll}1 & 0\end{array}\right) \quad$ wit h $M^{\lambda}\left(\pi_{\tilde{\alpha}}\right)=1-M^{\lambda}\left(u_{\tilde{\alpha}}\right)-M^{\lambda}\left(v_{\tilde{\alpha}}\right)$, we observe that,

$$
\begin{aligned}
S_{\mathrm{New}}(\tilde{\alpha})= & M^{\lambda}\left(u_{\tilde{\alpha}}\right)-M^{\lambda}\left(v_{\tilde{\alpha}}\right) \\
& +(2 \theta-1)\left(1-M^{\lambda}\left(u_{\tilde{\alpha}}\right)-M^{\lambda}\left(v_{\tilde{\alpha}}\right)\right) .
\end{aligned}
$$

Notably,

$0 \leq \theta \leq 1,1-M^{\lambda}\left(u_{\tilde{\alpha}}\right)-M^{\lambda}\left(v_{\tilde{\alpha}}\right) \geq 0$,

$M^{\lambda}\left(v_{\tilde{\alpha}}\right) \geq 0$.

Hence,

$$
\begin{aligned}
& S_{\mathrm{New}}(\tilde{\alpha}) \leq M^{\lambda}\left(u_{\tilde{\alpha}}\right)-M^{\lambda}\left(v_{\tilde{\alpha}}\right) \\
& \quad+\left(1-M^{\lambda}\left(u_{\tilde{\alpha}}\right)-M^{\lambda}\left(v_{\tilde{\alpha}}\right)\right)=1-2 M^{\lambda}\left(v_{\tilde{\alpha}}\right) \leq 1 .
\end{aligned}
$$

However,

$$
\begin{aligned}
S_{\mathrm{New}}(\tilde{\alpha}) & =M^{\lambda}\left(u_{\tilde{\alpha}}\right)-M^{\lambda}\left(v_{\tilde{\alpha}}\right)+(2 \theta-1)\left(1-M^{\lambda}\left(u_{\tilde{\alpha}}\right)-M^{\lambda}\left(v_{\tilde{\alpha}}\right)\right) \\
& =2 M^{\lambda}\left(u_{\tilde{\alpha}}\right)+2 \theta M^{\lambda}\left(\pi_{\tilde{\alpha}}\right)-1 .
\end{aligned}
$$

Considering that,

$M^{\lambda}\left(u_{\tilde{\alpha}}\right) \geq 0, M^{\lambda}\left(\pi_{\tilde{\alpha}}\right) \geq 0$,

$\theta \geq 0$,

we obtain,

$S_{\mathrm{New}}(\tilde{\alpha}) \geq-1$.

This result establishes

$-1 \leq S_{\mathrm{New}}(\tilde{\alpha}) \leq 1$ for any $\lambda, \theta \in[0,1]$.

2. Combining Eq. (10) with

$$
\begin{aligned}
& M^{\lambda}\left(u_{\tilde{\alpha}}\right)=a+\lambda(b-a), M^{\lambda}\left(v_{\tilde{\alpha}}\right)=c+(1-\lambda)(d-c), \\
& M^{\lambda}\left(\pi_{\tilde{\alpha}}\right)=\lambda(1-b-c)+(1-\lambda)(1-a-d),
\end{aligned}
$$

we can observe

$$
\begin{aligned}
S_{\mathrm{New}}(\tilde{\alpha})= & 2 a-1+2 \lambda(b-a)+2 \theta(1-a-d) \\
& +2 \theta \lambda(a+d-b-c) .
\end{aligned}
$$

If $S_{\mathrm{New}}(\tilde{\alpha})=1$ for any $\lambda, \theta \in[0,1]$ according to Eq. (12), we can obtain

$2 a-1=1,2(b-a)=0$,

$2(1-a-d)=0$,

$2(a+d-b-c)=0$.
Then,

$\tilde{\alpha}=([1,1],[0,0])$.

However, if $\tilde{\alpha}=([1,1],[0,0])$, then we can easily observe $S_{\text {New }}(\tilde{\alpha})=1$ for any $\lambda, \theta \in[0,1]$.

Hence,

$S_{\mathrm{New}}(\tilde{\alpha})=1 \Leftrightarrow \tilde{\alpha}=([1,1],[0,0])$ for any $\lambda, \theta \in[0,1]$.

3. If $S_{\mathrm{New}}(\tilde{\alpha})=-1$ for any $\lambda, \theta \in[0,1]$. Using Eq. (12), we can obtain

$2 a-1=-1,2(b-a)=0$,

$2(1-a-d)=0$

$2(a+d-b-c)=0$.

Thus,

$\tilde{\alpha}=([0,0],[1,1])$.

However, if $\tilde{\alpha}=([0,0],[1,1])$, then we can easily observe $S_{\text {New }}(\tilde{\alpha})=-1$ for any $\lambda, \theta \in[0,1]$.

Hence,

$S_{\text {New }}(\tilde{\alpha})=-1 \Leftrightarrow \tilde{\alpha}=([0,0],[1,1])$ for any $\lambda, \theta \in[0,1]$.

Property 2 Let $\tilde{\alpha}=\left(\left[a_{1}, b_{1}\right],\left[c_{1}, d_{1}\right]\right) \quad$ and $\tilde{\beta}=\left(\left[a_{2}, b_{2}\right],\left[c_{2}, d_{2}\right]\right)$ be two IVIFNs. If $a_{1} \geq a_{2}, b_{1} \geq b_{2}$ and $c_{1} \leq c_{2}, d_{1} \leq d_{2}$, then $S_{\mathrm{New}}(\tilde{\alpha}) \geq S_{\mathrm{New}}(\tilde{\beta})$.

Proof According to Eq. (10), by taking the partial derivative of $S_{\mathrm{New}}(\tilde{\alpha})$ with respect to $a, b, c$, and $d$, we can obtain,

$\frac{\partial S_{\mathrm{New}}(\tilde{\alpha})}{\partial a}=2(1-\lambda)(1-\theta), \quad \frac{\partial S_{\mathrm{New}}(\tilde{\alpha})}{\partial b}=2 \lambda(1-\theta)$,

$\frac{\partial S_{\mathrm{New}}(\tilde{\alpha})}{\partial c}=-2 \lambda \theta$, and $\frac{\partial S_{\mathrm{New}}(\tilde{\alpha})}{\partial d}=-2(1-\lambda) \theta$.

Notably, $0 \leq \lambda \leq 1$, and $0 \leq \theta \leq 1$. Hence,

$\frac{\partial S_{\mathrm{New}}(\tilde{\alpha})}{\partial a} \geq 0$,

$\frac{\partial S_{\mathrm{New}}(\tilde{\alpha})}{\partial b} \geq 0$,

$\frac{\partial S_{\mathrm{New}}(\tilde{\alpha})}{\partial c} \leq 0$,

$\frac{\partial S_{\mathrm{New}}(\tilde{\alpha})}{\partial d} \leq 0$.

Then, $S_{\mathrm{New}}(\tilde{\alpha})$ on $a$ and $b$ monotonously increases; $S_{\mathrm{New}}(\tilde{\alpha})$ on $c$ and $d$ monotonously decreases.

Therefore, if

$a_{1} \geq a_{2}, b_{1} \geq b_{2}, c_{1} \leq c_{2}$, and $d_{1} \leq d_{2}$, 
then,

$S_{\mathrm{New}}(\tilde{\alpha}) \geq S_{\mathrm{New}}(\tilde{\beta})$.

Property 3 Let $\tilde{\alpha}=\left(\left[a_{1}, b_{1}\right],\left[c_{1}, d_{1}\right]\right) \quad$ and $\tilde{\beta}=\left(\left[a_{2}, b_{2}\right],\left[c_{2}, d_{2}\right]\right)$ be two IVIFNs. For any $\lambda, \theta \in[0,1]$, $S_{\mathrm{New}}(\tilde{\alpha})=S_{\mathrm{New}}(\tilde{\beta})$ if and only if $\tilde{\alpha}=\tilde{\beta}$.

Proof According to Eq.(10), we obtain,

$$
\begin{aligned}
& S_{\mathrm{New}}(\tilde{\alpha})= 2 a_{1}-1+2 \lambda\left(b_{1}-a_{1}\right)+2 \theta\left(1-a_{1}-d_{1}\right) \\
&+2 \theta \lambda\left(a_{1}+d_{1}-b_{1}-c_{1}\right), \\
& S_{\mathrm{New}}(\tilde{\beta})= 2 a_{2}-1+2 \lambda\left(b_{2}-a_{2}\right)+2 \theta\left(1-a_{2}-d_{2}\right) \\
&+2 \theta \lambda\left(a_{2}+d_{2}-b_{2}-c_{2}\right) . \\
& \\
& \text { If } S_{\mathrm{New}}(\tilde{\alpha})=S_{\mathrm{New}}(\tilde{\beta}) \text { for any } \lambda, \theta \in[0,1], \\
& \text { then, we obtain }
\end{aligned}
$$

$2 a_{1}-1=2 a_{2}-1,2\left(b_{1}-a_{1}\right)=2\left(b_{2}-a_{2}\right)$,

$2\left(1-a_{1}-d_{1}\right)=2\left(1-a_{2}-d_{2}\right)$,

$2\left(a_{1}+d_{1}-b_{1}-c_{1}\right)=2\left(a_{2}+d_{2}-b_{2}-c_{2}\right)$.

Evidently,

$a_{1}=a_{2}, b_{1}=b_{2}, c_{1}=c_{2}$,

$d_{1}=d_{2}$.

Then,

$\tilde{\alpha}_{1}=\tilde{\alpha}_{2}$.

However, if $\tilde{\alpha}=\tilde{\beta}$, then evidently, $S_{\text {New }}(\tilde{\alpha})=S_{\text {New }}(\tilde{\beta})$ for any $\lambda, \theta \in[0,1]$. Hence,

$S_{\mathrm{New}}(\tilde{\alpha})=S_{\mathrm{New}}(\tilde{\beta}) \Leftrightarrow \tilde{\alpha}=\tilde{\beta}$ for any $\lambda, \theta \in[0,1]$.

\section{MCDM in interval-valued intuitionistic fuzzy environments}

In this section, an MCDM problem in an interval-valued intuitionistic fuzzy environment is presented to illustrate the feasibility and effectiveness of the proposed method.

Let $A=\left\{A_{1}, A_{2}, \ldots, A_{n}\right\}$ be a set of alternatives, and let $G=\left\{G_{1}, G_{2}, \ldots, G_{m}\right\}$ be a set of criteria. Assume that the weight of criteria $G_{j}, j=1,2, \ldots, m$ is provided by the decision-makers and denoted as $w_{j}, w_{j} \in[0,1]$, and $\sum_{j=1}^{m} w_{j}=1$. The evaluation of alternatives $A_{i}$ with respect to criterion $G_{j}$ is provided by IVIFNs and denoted as $\tilde{r}_{i j}=\left(\left[a_{i j}^{\prime}, b_{i j}^{\prime}\right],\left[c_{i j}^{\prime}, d_{i j}^{\prime}\right]\right), i=1,2, \ldots, n . j=1,2, \ldots, m$, which indicates the degree of alternative $A_{i}$ that satisfies or does not satisfy criterion $G_{j}$. Therefore, the decision matrix $D=\left(\tilde{r}_{i j}\right)_{n \times m}$ is obtained, which is expressed by IVIFNs.
In summary, the MCDM procedure is designed to determine the best alternative. This procedure is discussed in the following steps.

Step 1: Normalize the IVIFN decision matrix

Using the formulae introduced in Ye [29], the normalized IVIFN decision matrix $\bar{D}=\left(\tilde{\alpha}_{i j}\right)_{n \times m}$ is obtained, where $\tilde{\alpha}_{i j}=\left(\left[a_{i j}, b_{i j}\right],\left[c_{i j}, d_{i j}\right]\right)$ and $w=\left(w_{1}, w_{2}, \ldots, w_{m}\right)^{T}$ are the weight vector of the criteria.

Step 2: Calculate the weighted comprehensive score values

Choose the attitude parameter values $\lambda$ and $\theta$ according to the decision-makers' risk attitudes. Then, the weighted comprehensive score value $S_{i}$ of alternatives $A_{i}, i=1,2, \ldots, n$ can be obtained by the following formula:

$$
\begin{aligned}
S_{i}\left(\tilde{\alpha}_{i 1}, \tilde{\alpha}_{i 2}, \ldots, \tilde{\alpha}_{i m}\right)= & w_{1} S_{\mathrm{New}}\left(\tilde{\alpha}_{i 1}\right)+w_{2} S_{\mathrm{New}}\left(\tilde{\alpha}_{i 2}\right) \\
& +\cdots+w_{m} S_{\mathrm{New}}\left(\tilde{\alpha}_{i m}\right) .
\end{aligned}
$$

Step 3: Obtain the ranking of the alternatives

The ranking of alternatives $A_{i}, i=1,2, \ldots, n$ can be obtained based on the weighted comprehensive score values $S_{i}, i=1,2, \ldots, n$.

\section{Illustrative example}

In this section, we present the application of the proposed decision-making method through a practical example introduced in [49]. Comparative analyses of this method are also conducted to show the effectiveness of the proposed method.

A panel has four possible alternatives for company investment: (1) $A_{1}$ is a car company, (2) $A_{2}$ is a food company, (3) $A_{3}$ is a computer company, and (4) $A_{4}$ is an arms company. The investment company will make a decision according to five criteria: (1) $G_{1}$ is the productivity, (2) $G_{2}$ is the technological innovation capability, (3) $G_{3}$ is the marketing capability, (4) $G_{4}$ is the management, and (5) $G_{5}$ is the risk avoidance. The criteria are independent, and the criterion weights are provided by the decision-makers as follows: $w=(0.2,0.3,0.15,0.1,0.25)^{T}$. Since the available investment companies involve several different industries, the decision-makers may not have sufficient knowledge to provide the membership degree and non-membership degree of the admissible alternatives evaluated under the given several attributes as crisp values. Therefore, their ranges can be provided. Using IVIFNs to express decision-makers' evaluation information is considered the most effective method. The evaluation information is included in the IVIFN decision matrix $\bar{D}=\left(\tilde{\alpha}_{i j}\right)_{4 \times 5}$, which is a normalized one. 


$$
\bar{D}=\left(\begin{array}{llll}
([0.4,0.5],[0.1,0.3]) & ([0.5,0.6],[0.1,0.2])([0.3,0.4],[0.2,0.3]) & ([0.7,0.8],[0.1,0.2])([0.5,0.6],[0.1,0.2]) \\
([0.5,0.6],[0.1,0.2]) & ([0.3,0.4],[0.1,0.3])([0.7,0.8],[0.1,0.2]) & ([0.3,0.4],[0.3,0.4])([0.4,0.5],[0.1,0.2]) \\
([0.6,0.7],[0.1,0.2]) & ([0.7,0.8],[0.1,0.2])([0.5,0.6],[0.3,0.4]) & ([0.4,0.5],[0.3,0.4])([0.3,0.5],[0.3,0.4]) \\
([0.5,0.6],[0.2,0.3]) & ([0.4,0.5],[0.3,0.4])([0.6,0.7],[0.2,0.3]) & ([0.6,0.7],[0.2,0.3])([0.6,0.7],[0.1,0.3])
\end{array}\right)
$$

\section{Process developed using the proposed method}

To select the best potential company in which to invest, the decision-making process is developed through the following steps.

Step 1: Normalize the IVIFN decision matrix

Table 1 The weighted comprehensive score values with different attitude parameters

\begin{tabular}{ll}
\hline Attitude parameters & Weighted comprehensive score value \\
\hline$\lambda=\theta=\frac{1}{3}$ & $S_{\text {New }}\left(\tilde{\alpha}_{1}\right)=0.2078, S_{\text {New }}\left(\tilde{\alpha}_{2}\right)=0.14$ \\
& $S_{\text {New }}\left(\tilde{\alpha}_{3}\right)=0.2378, S_{\text {New }}\left(\tilde{\alpha}_{4}\right)=0.2122$ \\
$\lambda=\theta=\frac{1}{2}$ & $S_{\text {New }}\left(\tilde{\alpha}_{1}\right)=0.345, S_{\text {New }}\left(\tilde{\alpha}_{2}\right)=0.29$ \\
& $S_{\text {New }}\left(\tilde{\alpha}_{3}\right)=0.3325, S_{\text {New }}\left(\tilde{\alpha}_{4}\right)=0.3025$ \\
$\lambda=\theta=\frac{2}{3}$ & $S_{\text {New }}\left(\tilde{\alpha}_{1}\right)=0.4844, S_{\text {New }}\left(\tilde{\alpha}_{2}\right)=0.4433$ \\
& $S_{\text {New }}\left(\tilde{\alpha}_{3}\right)=0.4244, S_{\text {New }}\left(\tilde{\alpha}_{4}\right)=0.3956$ \\
\hline
\end{tabular}

The normalized IVIFN decision matrix is provided by the decision-makers and shown in decision matrix $\bar{D}=\left(\tilde{\alpha}_{i j}\right)_{4 \times 5}$.

Step 2: Calculate the weighted comprehensive score values

Select the attitude parameter values $\lambda, \theta \in[0,1]$ according to the decision-makers' risk attitudes. Then, the weighted comprehensive score values of alternatives $A_{i}, i=1,2,3,4$ can be obtained according to Eq. (13). The computing results are shown in Table 1.

Step 3: Obtain the ranking of the alternatives

According to the weighted comprehensive score values shown in Step 2, the alternatives are ranked as follows: (1) if $\lambda=\theta=\frac{1}{3}$, then $A_{3}>A_{4}>A_{1}>A_{2}$, which implies that a computer company is the best alternative for the company to invest in; (2) if $\lambda=\theta=\frac{1}{2}$, then $A_{1}>A_{3}>A_{4}>A_{2}$, which implies that a car company is the best alternative for the company to invest in; and (3) if $\lambda=\theta=\frac{2}{3}$, then $A_{1}>A_{2}>$ $A_{3}>A_{4}$, and a car company is the best alternative for the company to invest in.
Table 2 Ranking results obtained from different methods

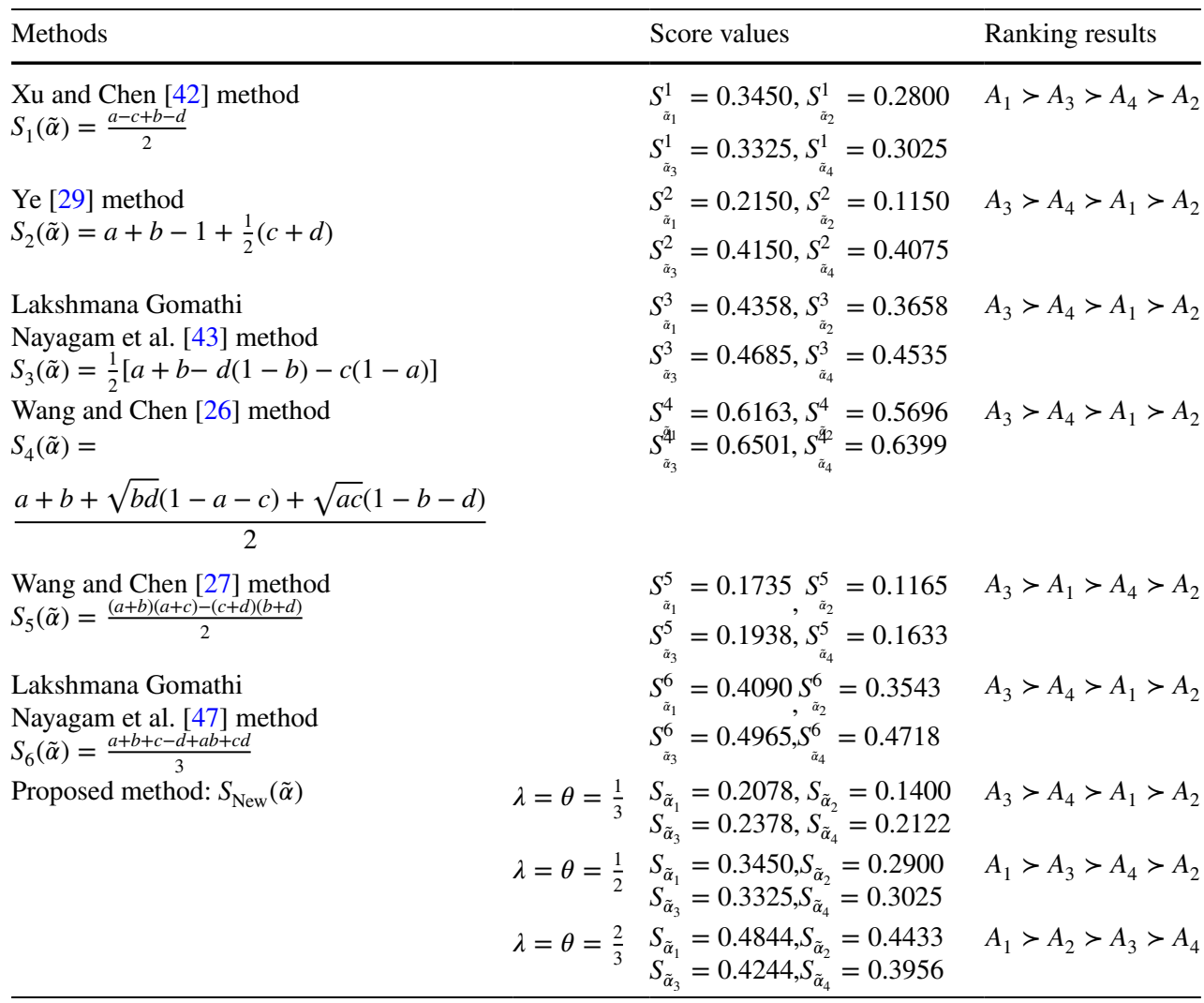




\section{Comparative study and discussion}

To validate the feasibility of the proposed approach, a comparative study with other approaches is conducted on the basis of the identical illustrative example introduced before "Process developed using the proposed method". To compare these approaches, the ranking results obtained from the different methods are listed in Table 2.

Table 2 shows that the ranking results derived from the approach of $\mathrm{Xu}$ and Chen [42] are identical to the ranking results of the proposed approach when $\lambda=\theta=\frac{1}{2}$. The ranking results derived from the approaches of Ye [29], Lakshmana Gomathi Nayagam et al. [43], Wang and Chen [26], and Lakshmana Gomathi Nayagam et al. [47] are identical to the proposed approach when $\lambda=\theta=\frac{1}{3}$. When $\lambda=\theta=\frac{2}{3}$, the method of Wang and Chen [27] and the proposed method are different from the other methods. However, the best alternative derived from Wang and Chen [27] is identical to those from Ye [29], Lakshmana Gomathi Nayagam et al. [43], Wang and Chen [26], and Nayagam et al. [47]. The best alternative is derived from $\mathrm{Xu}$ and Chen [42] method and the proposed approach when $\lambda=\theta=\frac{1}{2}$ and $\lambda=\theta=\frac{1}{3}$ are the same, thereby indicating that the proposed approach is effective. However, the results derived from the proposed method vary when decision-makers have different risk attitudes. Identical rankings may be derived using different approaches because when the value is set to $\lambda=\theta=\frac{1}{2}$ in the proposed method, the ranking index is equivalent to that in $\mathrm{Xu}$ and Chen [42] method. The same ranking index results in identical outcomes. The ranking indexes are different when the value is set to $\lambda=\theta=\frac{1}{3}$ in the proposed method compared with the ranking indexes of Ye [29], Lakshmana Gomathi Nayagam et al. [43], Wang and Chen [26], and Lakshmana Gomathi Nayagam et al. [47]. These approaches derive the rankings of the alternatives on the basis of the score values of IVIFNs. Therefore, the index of the score values of IVIFNs may result in identical ranking results.

In addition, Table 2 shows that the ranking results are different depending on decision-makers' risk attitudes. Risk-averse decision-makers believe that $A_{3}>A_{4}>A_{1}>A_{2}$. Risk-neutral decision-makers believe that $A_{1}>A_{3}>A_{4}>A_{2}$, while risk-seeking decision-makers believe that $A_{1}>A_{2}>A_{3}>A_{4}$. The risk attitudes of the decision-makers are confirmed to affect the decision results in the decision-making process. Based on this fact, the risk parameter and score function must be combined, and the proposed method in this paper has a wide practical application.

The comparative analysis indicates that the MCDM approach proposed in this study has the following advantages over the other approaches,
1. Based on the membership degree, non-membership degree, hesitancy degree, and decision-makers' risk attitudes, a new score function has been proposed to reflect that the decision results are highly affected by the decision-makers' risk attitudes.

2. The proposed new score function is equivalent to $\mathrm{Xu}$ and Chen [42] method when the decision-makers' risk attitudes are set to certain values. This finding confirms that $\mathrm{Xu}$ and Chen [42] method is a special case of the proposed method, and the proposed method has a wide practical application.

\section{Conclusions}

To examine decision-makers' mentality, two mentality parameters are introduced in this paper. First, a new score function of IVIFNs is proposed that uses the introduced mentality parameters. Second, some properties of the proposed score function of IVIFNs are discussed to confirm the score function's reasonability and effectiveness. Furthermore, the weighted comprehensive score value of IVIFNs is proposed, and an MCDM problem is developed in an interval-valued intuitionistic fuzzy environment. Finally, a numerical example and comparative analyses are provided to illustrate the feasibility and effectiveness of the proposed method.

This study provides several substantial contributions to MCDM problems, which are summarized as follows. (1) A new score function has been proposed to reflect decision-makers' risk attitudes. The risk parameters of decision-makers are associated with the membership degree, non-membership degree, and hesitancy degree of IVIFNs. (2) The proposed MCDM method is more widely used than $\mathrm{Xu}$ and Chen [42] method in practice, as the latter is a special case of the proposed method. In the future, MCDM with large-scale social networks is worth examining, and the complex practical problems should be further studied.

Acknowledgements The authors thank the anonymous reviewers and the editor for their insightful and constructive comments and suggestions that have led to an improved version of this paper. This work was supported by the National Natural Science Foundation of China (No. 61866006), Guangxi innovation-driven development of special funds project (gui ke AA17204091), the Natural Science Foundation of Guangxi (No. AB17292095), the Research Funds for the Guangxi University Xingjian College of Science and Liberal Arts (No. Y2018ZKT01) and Promotion project of Middle-aged and Young Teachers' Basic Scientific Research Ability in Universities of Guangxi (No. 2019KY0963).

Open Access This article is licensed under a Creative Commons Attribution 4.0 International License, which permits use, sharing, adaptation, distribution and reproduction in any medium or format, as long as you give appropriate credit to the original author(s) and the source, 
provide a link to the Creative Commons licence, and indicate if changes were made. The images or other third party material in this article are included in the article's Creative Commons licence, unless indicated otherwise in a credit line to the material. If material is not included in the article's Creative Commons licence and your intended use is not permitted by statutory regulation or exceeds the permitted use, you will need to obtain permission directly from the copyright holder. To view a copy of this licence, visit http://creativecommons.org/licenses/by/4.0/.

\section{References}

1. Atanassov K (1986) Intuitionistic fuzzy sets. Fuzzy Sets Syst 20:87-96

2. Zadeh LA (1965) Fuzzy sets. Inf Control 8:338-353

3. Alcantud JCR, Khameneh AZ, Kilicman A (2019) Aggregation of infinite chains of intuitionistic fuzzy sets and their application to choices with temporal intuitionistic fuzzy information. Inf Sci. https://doi.org/10.1016/j.ins.2019.1012.1008

4. Ngan RT, Son LH, Ali M, Tamir DE, Rishe ND, Kandel A (2019) Representing complex intuitionistic fuzzy set by quaternion numbers and applications to decision making. Appl Soft Comput 33:105961

5. Zhang K, Huang YD, Yuan X, Zhao CX (2019) Infrared and visible image fusion based on intuitionistic fuzzy sets. Infrared Phys Technol 1:103124

6. Zheng M, Liu Y (2019) Multiple-rules reasoning based on Triple I method on Atanassov's intuitionistic fuzzy sets. Int J Approx Reason 113:196-206

7. Melliani S, Castillo O (2019) Recent advances in intuitionistic fuzzy logic systems. Springer, Berlin, pp 291-299

8. Roeva O, Michalikova A (2013) Generalized net model of intuitionistic fuzzy logic control of genetic algorithm parameters. In: 9th Int. Workshop on IFSs, Banská Bystrica, 8 October 2013 Notes on Intuitionistic Fuzzy Sets, pp71-76

9. Atanassov K, Sotirov S (2020) Intuitionistic fuzzy neural networks with interval valued intuitionistic fuzzy conditions, Intuitionistic and Type-2 fuzzy logic enhancements in neural and optimization algorithms: theory and applications. Springer, Berlin, pp 99-106

10. Atanassov K, Gargov G (1989) Interval valued intuitionistic fuzzy sets. Fuzzy Sets Syst 31:343-349

11. Cheng SH (2018) Autocratic multiattribute group decision making for hotel location selection based on interval-valued intuitionistic fuzzy sets. Inf Sci 427:77-87

12. Alaoui ME, Ben-azza H, Yassini KE (2019) Achieving consensus in interval valued intuitionistic fuzzy environment. Procedia Comput Sci 148:218-225

13. Zhang ZM, Wu C, Pedrycz W (2019) A novel group decisionmaking method for interval-valued intuitionistic multiplicative preference relations. IEEE Trans Fuzzy Syst. https://doi. org/10.1109/TFUZZ.2019.2922917

14. Couso I, Bustince H (2019) Three categories of set-valued generalizations from fuzzy sets to interval-valued and atanassov intuitionistic fuzzy sets. IEEE Trans Fuzzy Syst 27:362-370

15. Wang CY, Chen SM (2017) Multiple attribute decision making based on interval-valued intuitionistic fuzzy sets, linear programming methodology, and the extended TOPSIS method. Inf Sci 397-398:155-167

16. Deveci M, Öner SC, Canitez F, Öner M (2019) Evaluation of service quality in public bus transportation using intervalvalued intuitionistic fuzzy QFD methodology. Res Transp Bus Manag. https://doi.org/10.1016/j.rtbm.2019.100387
17. Wang F, Wan SP (2020) Possibility degree and divergence degree based method for interval-valued intuitionistic fuzzy multi-attribute group decision making. Expert Syst Appl 141:112929

18. Mishra AR, Rani P, Mardani A, Pardasani KR, Govindan K, Alrasheedi M (2020) Healthcare evaluation in hazardous waste recycling using novel interval-valued intuitionistic fuzzy information based on complex proportional assessment method. Comput Ind Eng 139:106140

19. Abdullah L, Zulkifli N, Liao H, Herrera-Viedma E, Al-Barakati A (2019) An interval-valued intuitionistic fuzzy DEMATEL method combined with Choquet integral for sustainable solid waste management. Eng Appl Artif Intell 82:207-215

20. Wang Y, Shi Y (2019) Measuring the service quality of urban rail transit based on interval-valued intuitionistic fuzzy model. KSCE J Civil Eng. https://doi.org/10.1007/s12205-12020-10937-x

21. Yue C (2019) An interval-valued intuitionistic fuzzy projectionbased approach and application to evaluating knowledge transfer effectiveness. Neural Comput Appl 31:7685-7706

22. Deveci M, Öner SC, Canitez F, Öner M (2019) Evaluation of service quality in public bus transportation using interval-valued intuitionistic fuzzy QFD methodology. Res Transp Bus Manag 33:100387

23. Zhang W, Ding J, Wang Y, Zhang S, Xiong Z (2019) Multi-perspective collaborative scheduling using extended genetic algorithm with interval-valued intuitionistic fuzzy entropy weight method. J Manuf Syst 53:249-260

24. Wang LE, Liu HC, Quan MY (2016) Evaluating the risk of failure modes with a hybrid MCDM model under interval-valued intuitionistic fuzzy environments. Comput Ind Eng 102:175-185

25. Long S, Geng S (2015) Decision framework of photovoltaic module selection under interval-valued intuitionistic fuzzy environment. Energy Convers Manage 106:1242-1250

26. Wang CY, Chen SM (2017) An improved multiattribute decision making method based on new score function of interval-valued intuitionistic fuzzy values and linear programming methodology. Inf Sci 411:176-184

27. Wang CY, Chen SM (2018) A new multiple attribute decision making method based on linear programming methodology and novel score function and novel accuracy function of interval-valued intuitionistic fuzzy values. Inf Sci 438:145-155

28. Wan SP, Wang F, Dong JY (2017) Additive consistent intervalvalued Atanassov intuitionistic fuzzy preference relation and likelihood comparison algorithm based group decision making. Eur J Oper Res 263:571-582

29. Ye J (2009) Multicriteria fuzzy decision-making method based on a novel accuracy function under interval-valued intuitionistic fuzzy environment. Expert Syst Appl 36:6899-6902

30. Kaur A, Kumar A (2019) Commentary on "A geometric approach for ranking interval-valued intuitionistic fuzzy numbers with an application to group decision-making". Comput Ind Eng 135:314-316

31. Liu Y, Jiang W (2019) A new distance measure of interval-valued intuitionistic fuzzy sets and its application in decision making. Soft Comput. https://doi.org/10.1007/s00500-00019-04332-00505

32. Wei AP, Li DF, Jiang BQ, Lin PP (2019) The novel generalized exponential entropy for intuitionistic fuzzy sets and interval valued intuitionistic fuzzy sets. Int J Fuzzy Syst 21:2327-2339

33. Yue C (2016) A geometric approach for ranking interval-valued intuitionistic fuzzy numbers with an application to group decisionmaking. Comput Ind Eng 102:233-245

34. Gupta P, Mehlawat MK, Grover N, Pedrycz W (2018) Multiattribute group decision making based on extended TOPSIS method under interval-valued intuitionistic fuzzy environment. Appl Soft Comput 69:554-567 
35. Hajek P, Froelich W (2019) Integrating TOPSIS with intervalvalued intuitionistic fuzzy cognitive maps for effective group decision making. Inf Sci 485:394-412

36. Zeng SZ, Chen SM, Fan KY (2020) Interval-valued intuitionistic fuzzy multiple attribute decision making based on nonlinear programming methodology and TOPSIS method. Inf Sci 506:424-442

37. Li J, Wang J, Hu J (2018) Consensus building for hesitant fuzzy preference relations with multiplicative consistency. Comput Ind Eng 128:387-400

38. Chen SM, Fan KY (2019) Multiattribute decision making based on probability density functions and the variances and standard deviations of largest ranges of evaluating interval-valued intuitionistic fuzzy values. Inf Sci 490:329-343

39. Zhang W, Ding J, Wang Y, Zhang S, Xiong Z (2019) Multiperspective collaborative scheduling using extended genetic algorithm with interval-valued intuitionistic fuzzy entropy weight method. Sens Actuators A. https://doi.org/10.1016/j. sna.2019.111670

40. Wan SP, Wang F, Dong JY (2018) A group decision-making method considering both the group consensus and multiplicative consistency of interval-valued intuitionistic fuzzy preference relations. Inf Sci 466:109-128

41. Tang J, Meng FY, Zhang YL (2018) Decision making with interval-valued intuitionistic fuzzy preference relations based on additive consistency analysis. Inf Sci 467:115-134

42. Xu ZS, Chen J (2007) Approach to group decision making based on interval-valued intuitionistic judgment matrices. Syst Eng Theory Pract 27:126-133
43. Lakshmana Gomathi Nayagam V, Muralikrishnan S, Sivaraman G (2011) Multi-criteria decision-making method based on interval-valued intuitionistic fuzzy sets. Expert Syst Appl 38(2011):1464-1467

44. Chen SM, Huang ZC (2017) Multiattribute decision making based on interval-valued intuitionistic fuzzy values and linear programming methodology. Inf Sci 381:341-351

45. Nguyen H (2019) A generalized p-norm knowledge-based score function for interval-valued intuitionistic fuzzy set in decision making. IEEE Trans Fuzzy Syst. https://doi.org/10.1109/TFUZZ .2019 .2907068

46. Wang JQ, Li KJ, Zhang HY (2012) Interval-valued intuitionistic fuzzy multi-criteria decision-making approach based on prospect score function. Knowl-Based Syst 27:119-125

47. Lakshmana Gomathi Nayagam V, Jeevaraj S, Dhanasekaran P (2017) An intuitionistic fuzzy multi-criteria decision-making method based on non-hesitance score for interval-valued intuitionistic fuzzy sets. Soft Comput 21(2017):7077-7082

48. Xu ZS (2007) Methods for aggregating interval-valued intuitionistic fuzzy information and their application to decision making. Control Decis 22:215-219

49. Herrera F, Herrera-Viedma E (2000) Linguistic decision analysis: steps for solving decision problems under linguistic information. Fuzzy Sets Syst 115:67-82

Publisher's Note Springer Nature remains neutral with regard to jurisdictional claims in published maps and institutional affiliations. 